\title{
COMPETITIVE EQUILIBRIA IN GENERAL CHOICE SPACES
}

\author{
Darrell Duffie \\ Graduate School of Business \\ Stanford University \\ Stanford, CA, 94305
}

\begin{abstract}
This paper primarily demonstrates the existence of Arrow-Debreu equilibria in a general class of topological vector spaces of commodity bundles. Two conditions based on production possibilities, preferences, and the topological nature of bounded sets are shown to substitute, in any locally convex space, for the advantages of the Euclidean topology. Examples fulfilling these conditions are supplied. The approach is that of Bewley, demonstrating equilibria on finite-dimensional sub-economies and establishing a net of these equilibria that converges to an equilibrium on the whole commodity space. An example of equilibrium with a storage technology is given. An auxiliary result concerns the price support of efficient allocations.
\end{abstract}

This paper was distributed in draft in July 1983. A version appeared in a dissertation completed in the Engineering-Economic Systems Department at Stanford University. I am grateful for the comments of David Luenberger, David Kreps, Kenneth Arrow, Donald Brown, Andreu Mas-Colell, William Zame, and a referee. 


\section{Introduction}

This paper addresses the problem of existence of Walrasian equilibria, stated and solved first for the finite-dimensional case by Arrow and Debreu [1], and generalized to roughly its ultimate state in the setting of finitely many agents with complete preference relations by Debreu [6; Chapter 10] in 1962. In departing from a Euclidean setting for this problem, one neither gains at all in tractability nor suffers inordinately from any failure of geometric insights. In approaching this problem then, the objective will be to take Debreu's 1962 assumptions as given, and limit any additional assumptions to a minimum. As one might expect, the set of conditions stated by Debreu, extended to any locally convex choice space, falls short on two key topological points: the compactness of feasible allocations in the choice space and the compactness of the price "simplex" in the dual space. These points are shown to be surmountable by adding two corresponding topological assumptions that can be immediately fulfilled in many useful infinite-dimensional choice spaces, including any of those previously studied in the context of this problem, such as $R^{n}$, $L^{p}$ spaces, $\ell^{p}$ spaces, and any of the other conjugate Banach spaces. Other spaces encompassed in this paper include any of the semi-reflexive spaces or Montel spaces, for instance $C^{\infty}$ spaces of "smooth" functions (which are not normed spaces).

This is not to say, however, that all previous results are subsumed here. By insightfully posing smoothness conditions on agents' preferences, Andreu Mas-Colell [11] overcame these topological barriers by a different approach, as explained shortly. His work on an exchange economy in conjugate Banach lattices instigated this paper. Zame [18] has extended Mas-Colell's approach to production economies, but there is still a gap between the two basic approaches that remains as a challenge to be filled.

Why study infinite-dimensional spaces ? There are many applications whose natural setting involves "blends" of commodity characteristics, or uncertain states of the world governed by non-atomic probability measures, or dynamic models whose objects of choice are functions of time. Much new work in equilibrium theory addresses economies whose choice vectors are stochastic processes. In this and many other settings, agents' optimal plans can often be succinctly characterized via some form of control theory in infinite-dimensional function spaces. In short, a theory for general choice spaces is called for.

In equilibrium analysis one invariably wishes to select candidate price vectors from some compact convex set not including zero. The unit simplex for $R^{n}$ is just such a set for Euclidean analysis but its analogue in general dual spaces, for example positive price vectors of unit norm for normed spaces, need not be satisfactory. If the positive cone of the choice space has interior, however, the set of positive price vectors with value one, say, at a particular choice vector in the interior of this cone forms an appropriate "neo-simplex". This idea goes back as far as Debreu's papers of three decades ago [6; Chapters 5 and 7]. Of course one needn't limit oneself to the "original" positive cone of the choice space, particularly if it has no interior. MasColell, for example, constructs a cone with interior from the properties of agent's preferences; no vector in this cone can be split up among agents and subtracted from any efficient allocation so as to make each agent better off. Thus any efficient allocation can be supported in a "valuation equilibrium" [6; Ch.5] by a price vector 
with positive values on this cone by virtue of the separating hyperplane theorem. Such a cone is characterized as a "price block" in Section 3. Mas-Colell's result requires fairly weak restrictions on the choice space, but rather strong conditions on consumption sets - they must be the positive cone of the choice space.

The alternative taken here is to assemble the largest cone, say $\Gamma$, whose elements must have positive market value in any valuation equilibrium, and then to assume that $\Gamma$ has interior, fitting into its role as a price block, the pre-requisite for a neo-simplex. Debreu [6; Ch.10] constructed just such a cone to alleviate the free disposal assumption. Suppose, for example, that the production set contains a cone $A$. Then any valuation equilibrium places negative market values on $A$, for otherwise some firm has unbounded profits. Thus $-A \subset \Gamma$. Next, suppose $D$ is a cone, each of whose elements can be re-scaled and then shared among agents so that, added to their endowments, the resulting allocation Pareto dominates any feasible allocation. Then any element of $D$ must have a positive market value in any valuation equilibrium, and $D \subset \Gamma$. Further material can be added to $\Gamma$ as discussed in Section 3, in particular by augmenting the production set. How a production set might come to contain a cone with interior is also discussed there, with examples in Section 6. More work is called for on this last point.

The mechanics of demonstrating an equilibrium given a neo-simplex also differ from those of Mas-Colell, who finds a fixed point in the (utility simplex $\times$ price neo-simplex) space. Since functional utility representations of preferences in general choice spaces with production is not guaranteed, the fixed point here is in the (allocation $\times$ price neo-simplex) space. The stated philosophy of extending Euclidean insights to general choice spaces is actually put to work, following Truman Bewley's blueprint [4]. If Debreu's conditions apply to an economy $\mathcal{E}$ with a general choice space $L$, then they also apply to each of the "sub-economies" $\mathcal{E}_{\lambda}$ induced by a corresponding net $\left\{F_{\lambda}\right\}$ of finite-dimensional vector subspaces of $L$, directed so as to include any particular vector at some point in the net. Provided only that $L$ is locally convex, each $F_{\lambda}$ is equivalent topologically with a Euclidean space, whence an equilibrium for $\mathcal{E}_{\lambda}$ follows without any additional work. A cluster point of the resulting net of "sub-equilibria" exists and is an equilibrium for $\mathcal{E}$. The difficult competitive analysis, then, takes place in Euclidean space, where it is easy. Debreu's rather complicated proof is "boot-strapped" up to a general class of vector spaces.

The next section merely sets up the basic notation and definitions. The following one develops the notion of "price blocks". Section 4 has the main result, showing conditions ensuring the existence of quasi-equilibria with production in a general class of infinite-dimensional choice spaces. Section 5 shows the existence of price support for efficient allocations under less stringent conditions than those adopted for demonstrating equilibria. A simple example showing the new theory in action appears in Section 6. The final section contains a few general remarks. The Appendix extends several results on recession cones that Debreu [5] stated for Euclidean spaces.

\section{The Basic Equilibrium Problem}

In this section we briefly cover the notation and definitions for the competitive 
equilibrium problem, largely following Debreu [5]. Let $L$ be a vector (choice) space over the field of real numbers, with algebraic dual $L^{\prime}$, the space of linear functionals on $L$. We consider the following finite agent economy on $L$ :

$$
\mathcal{E}=\left(\left(X_{i}, \succeq_{i}\right) ;\left(Y_{j}\right) ;\left(\omega_{i}\right) ;\left(\theta_{i j}\right)\right),
$$

where $X_{i} \subset L$ are consumption sets, $\succeq_{i}$ are preferences (complete transitive binary orders) on $X_{i}, Y_{j} \subset L$ are production sets, $\omega_{i} \in L$ are endowments, and $\theta_{i j} \in[0,1]$ are firm shares, for all $i \in M=\{1, \ldots, m\}$ and all $j \in N=\{1, \ldots, n\}$. Of course $\sum_{i=1}^{m} \theta_{i j}=1$ for all $j \in N$. As usual, define $X=\sum_{i=1}^{m} X_{i}, Y=\sum_{j=1}^{n} Y_{j}$, and $\omega=\sum_{i=1}^{m} \omega_{i}$. An allocation is an $(m+n)$-tuple, $(x, y) \in L^{m+n}$, where

$$
x=\left(x_{1}, \ldots, x_{m}\right), x_{i} \in X_{i} \quad \forall i \in M
$$

and

$$
y=\left(y_{1}, \ldots, y_{n}\right), y_{j} \in Y_{j} \quad \forall j \in N .
$$

An allocation $(x, y)$ is feasible if $\sum_{i=1}^{m} x_{i}=\omega+\sum_{j=1}^{n} y_{j}$. An allocation $(x, y)$ is supported by $p \in L^{\prime}$ if $p \neq 0$,

$$
z \succeq_{i} x_{i} \quad \Rightarrow \quad p \cdot z \geq p \cdot x_{i} \quad \forall z \in X_{i} \quad \forall i \in M
$$

and

$$
p \cdot y_{j} \geq p \cdot z \quad \forall z \in Y_{j} \quad \forall j \in N,
$$

where $p \cdot z$ denotes $p$ evaluated at $z \in L$. If $p$ supports a feasible allocation $(x, y)$, then $(x, y, p)$ is a valuation equilibrium [6;Ch.5]. An allocation $(x, y)$ is budgetconstrained under $p \in L^{\prime}$ if

$$
p \cdot x_{i} \leq p \cdot\left[\omega_{i}+\sum_{j=1}^{n} \theta_{i j} y_{j}\right] \quad \forall i \in M
$$

For convenience, a budget-constrained valuation equilibrium is termed a compensated equilibrium, after an effectively equivalent concept in Arrow and Hahn [2;p.108]. If $(x, y)$ is a feasible allocation that is budget-constrained under $p \in L^{\prime}$, then $(x, y, p)$ is a quasi-equilibrium [6;Ch.10] provided for each $i \in M$,

$$
z \succ_{i} x_{i} \quad \Rightarrow \quad p \cdot z>p \cdot x_{i} \quad \forall i \in M
$$

or

$$
p \cdot z \geq p \cdot x_{i} \quad \forall z \in X_{i},
$$

where $\succ_{i}$ denotes the strict preference ordering induced by $\succeq_{i}$. An equilibrium is a quasi-equilibrium for which (2.2) holds for all $i \in M$.

Remark 2.1 The two concepts: compensated equilibrium and quasi-equilibrium are similar but independent; neither implies the other. Their close relationship can 
be deduced easily from Debreu's analysis $[5 ; 4.9(1)-(2)]$. One can see, for instance, that although Mas-Colell [11] defines a "quasi-equilibrium" to be what is termed a compensated equilibrium above, the two concepts are equivalent under his preference assumptions. The proof is not immediate, but is simple enough and left to the reader.

A feasible allocation $(x, y)$ is weakly efficient if there is no other feasible allocation $\left(x^{\prime}, y^{\prime}\right)$ satisfying $x_{i}^{\prime} \succ_{i} x_{i} \quad \forall i \in M$. A feasible allocation is efficient if there is no other feasible allocation $\left(x^{\prime}, y^{\prime}\right)$ satisfying $x_{i}^{\prime} \succeq_{i} x_{i} \quad \forall i \in M$, with some $k \in M$ such that $x_{k}^{\prime} \succ_{k} x_{k}$.

If $L$ is given a linear topology ${ }^{1}$, the topological dual of $L$, denoted $L^{*}$, is the subset of $L^{\prime}$ whose elements are continuous. We will limit our search for an equilibrium price functional to $L^{*}$ because of its convenient topological properties.

\section{Price Blocks}

This section illustrates how certain properties of the Euclidean positive cone (or orthant) that facilitate competitive analysis in $R^{n}$ can be recovered under the right conditions in general vector choice spaces with the following substitute for a positive cone. Throughout the remainder, some linear topology on $L$ is assumed. A convex cone $^{2} \Gamma \subset L$ is a price block for $\mathcal{E}$ provided it has interior and any price supported weakly efficient allocation can be supported by an element of $\Gamma^{\circ}$, the polar ${ }^{3}$ of $\Gamma$. If $\Gamma$ is a price block for $\mathcal{E}$, we can limit our search for an equilibrium price vector to $\Gamma^{\circ}$. This explains the term "price block": the hyperplanes supporting $\Gamma$ are blocked from intersecting an entire neighborhood contained by $\Gamma$, and thus from being "too steep" in any direction. In fact, since price vectors are subject to division by a strictly positive scalar without economic effect, we can restrict our search even further to $\Gamma_{\hat{y}}^{\circ}=\left\{p \in \Gamma^{\circ}: p \cdot \hat{y}=-1\right\}$, where $\hat{y}$ is an interior point of $\Gamma$. In Euclidean space space the "neo-simplex" $\Gamma_{\hat{y}}^{\circ}$ is a compact convex set, and substitutes for the classical price simplex $\Delta^{n-1}=\left\{p \in R_{+}^{n}: \sum_{\ell=1}^{n} p_{\ell}=1\right\}$. Later we will exploit the fact that the compactness of $\Gamma_{\hat{y}}^{\circ}$ extends, in an appropriate topology, to any locally convex space ${ }^{4}$. Then any net in $\Gamma_{\hat{y}}^{\circ}$ has a non-zero cluster point, which, with the

1 A topological vector space is a vector space that is also a topological space whose points are closed sets, and for which vector addition and scalar multiplication are continuous functions. A topology $\tau$ on a vector space $L$ is linear if $(L, \tau)$ is a topological vector space. It will always be implicit here that these are vector spaces over the scalar field of real numbers. This definition, due to Walter Rudin [8], ensures for convenience that any topological vector space is a Hausdorff space. A topological space is Hausdorff, or separated, or $T_{2}$, if every pair of distinct points have corresponding disjoint neighborhoods.

2 A subset $C$ of a vector space is a cone provided $\alpha C=C$ for any scalar $\alpha>0$.

3 The polar of any subset $A \subset L$ is the dual set $A^{\circ}=\left\{p \in L^{*}: p \cdot z \leq 1 \forall z \in A\right\}$. If $A$ is a cone, $A^{\circ}=\left\{p \in L^{*}: p \cdot z \leq 0 \forall z \in A\right\}$.

4 A locally convex space is a topological vector space with a neighborhood base of absolutely convex sets, that is, in which each open set contains a convex open set. Any vector space can be given a locally convex topology. 
right construction of the net, will turn out to be an equilibrium price vector. This same idea is used in Florenzano [7] and Mas-Colell [11], where $L$ is a Banach space and $\mathcal{E}$ is an exchange economy. Florenzano assumes that $L$ is also ordered ${ }^{5}$, and that its disposal cone has interior, thereby serving as a price block. Mas-Colell gives smoothness conditions on agents' preferences, called "properness", ensuring the existence of a price block, provided $L$ is a Banach lattice ${ }^{6}$ and additionally that each agent's consumption set is the positive cone. Mas-Colell's results are easily extended to locally convex vector lattices, by the following extension of properness. A preference relation $\succeq$ on a subset $X$ of a topological vector space $L$ is $v$-proper, for some non-zero vector $v \in L$, if there is a zero neighborhood $\mathcal{N}$ with the property: for any $x \in X, z \in L$, and scalar $\alpha>0$ such that $x-\alpha v+z \in X$, the relationship $x-\alpha v+z \succeq x$ implies that $z$ is not an element of $\alpha \mathcal{N}$. The intuition is that one cannot compensate a $v$-proper agent for a loss of $v$ by any vector that is "too small". For an exchange economy, this ensures that the set of vectors that can be shared among agents and added to any efficient allocation so as to yield a Pareto improvement is disjoint from a cone that includes as an interior point the sum across agents of the "properness" vectors $v$ [11; Prop. VII]. The resulting quasiequilibrium existence theorem is stated in Section 4.3.

One can also obtain a price block, perhaps, on the production side of the economy. Suppose some fixed "large" input vector $y^{I} \in L$ can be converted in production to any output vector $y^{O} \in \mathcal{N}$, where $\mathcal{N}$ is some sufficiently small neighborhood of zero. That is, suppose $y^{O}-y^{I} \in Y$ for all $y^{O} \in \mathcal{N}$, and furthermore that this production technology has constant returns to scale, implying that $\Gamma=\left\{\alpha\left(y^{O}-y^{I}\right) ; y^{O} \in \mathcal{N} ; \alpha \geq 0\right\} \subset Y$. Section 6 contains an example of such a technology. Clearly $\Gamma$ is a convex cone, and thus a price block. For if $p \in L^{\prime}$ supports any allocation, then $p \cdot y \leq 0$ for all $y \in \Gamma$, for otherwise profits would be unbounded, and thus $p \in \Gamma^{\circ}$.

Of course the existence of a price block contained by $Y$ need not arise from such a restrictive technology. The weakest condition on $Y$ ensuring that it contains a price block is that its recession cone $^{7}$, denoted $A(Y)$, has interior. This follows from the characterization of $A(Y)$ as the largest convex cone contained by $Y$, which is valid if $Y$ is convex and includes zero, but these are standard competitive assumptions. The assumption of free disposal, or $L_{+} \subset-Y$, of course satisfies the required assumption if $L_{+}$has interior, which is true for example in Euclidean space (Figure 2), and $\ell^{\infty}$ or $L^{\infty}(\mu)$ spaces, an observation made as early as 1953

5 A vector space $L$ is partially ordered by a convex cone $C$ by writing $x \geq y$ whenever $x-y \in C$. In this case $C$ is called the positive cone of $L$ and denoted $\bar{L}_{+}$.

6 An ordered vector space is a lattice (or Riesz space) provided the infimum of any two vectors under the ordering exists. See Schaefer [15]. For example, spaces of differentiable functions are not generally lattices. A locally convex vector lattice is a lattice that is a locally convex space in which the lattice decomposition $x \mapsto x^{+}$ is continuous. A Banach lattice is a locally convex vector lattice whose topology is given by a complete norm. Illustrative examples are the $L^{q}(\mu)$ spaces, $1 \leq q \leq \infty$.

7 The recession cone of a convex set $B \subset L$, denoted $A(B)$, is the set $\{z \in L$ : $\{z\}+B \subset B\}$. This is elaborated in the appendix. 
by Debreu [6;Ch.5].

Of course, it may be that $A(Y)$ has no interior, but even so, all is not lost! Following McKenzie [12,13] and Debreu [6;Ch.10], define an augmented production set to be any subset $\ddot{Y}$ of $L$ containing $Y$ and generating the same feasible allocations:

$$
(\{\omega\}+\ddot{Y}) \cap X=(\{\omega\}+Y) \cap X .
$$

For purposes of demonstrating an equilibrium, one can assume temporarily that the production set is $\ddot{Y}$. Any resulting equilibrium must also be an equilibrium for the economy whose production possibilities are given by the smaller production set $Y$. The trick, of course, is that one may be able to place enough potential production choices in $\ddot{Y}$ to ensure that $A(\ddot{Y})$ has interior. General conditions under which such an augmentation can be successful have not been studied.

There is yet a further weakening of the conditions ensuring the existence of a price block, again following related work by McKenzie and Debreu. First, let $\hat{X}_{i}$ denote the projection of the set of feasible allocations $(x, y) \in L^{m+n}$ into the $i$-th co-ordinate subspace. That is, $\hat{X}_{i}$ is the set of feasible allocations for agent $i$. Next, let $D$ be the smallest cone (that is, the intersection of all cones) in $L$ containing the preference generated set

$$
\mathcal{D}=\left\{z \in L: z=\sum_{i=1}^{m} z_{i} ; z_{i}+\omega_{i} \succ_{i} x_{i} \forall x_{i} \in \hat{X}_{i}, \forall i \in M\right\} .
$$

The set $\mathcal{D}$ is effectively the set of vectors that can be shared somehow among agents to strictly Pareto dominate any feasible allocation. For example, if $L$ is an ordered vector space and preferences are strictly monotonic ${ }^{8}$ then $L_{+} \subset D$. Roughly speaking, the production set $Y$ can also be augmented by $-D$ without changing the set of potential equilibria, a claim formalized by Debreu $[6 ;$ Ch.10]. Thus the convex cone $A(\ddot{Y})-D$ is also a price block provided it has interior, a much weaker condition than $\operatorname{int}(A(Y)) \neq \emptyset$.

If $L$ is a locally convex vector lattice and $X_{i}=L_{+}$for all $i$, still further weakening may be possible following the tack taken by Mas-Colell. A successful combination of the two approaches has yet to be demonstrated, however.

\section{Existence of Quasi-equilibria}

In this section we prove existence of quasi-equilibria with production in any locally convex choice space, under stated conditions. The approach is that of Bewley [4], establishing quasi-equilibria on a net of finite-dimensional subspaces of the choice space. These quasi-equilibria converge to a quasi-equilibrium on the whole space. The basic assumptions are those of Debreu [6;Ch.10], which is among the most general treatments of equilibrium existence for finite-dimensional competitive economies, plus two assumptions that meet our needs in the infinite-dimensional case.

${ }^{8}$ Let $W$ be a subset of an ordered vector space $L$ such that $W+L_{+}=W$. A preference relation $\succeq$ on $W$ is monotonic provided $w \in W$ implies $z \succeq w$ for all $z \geq w$, and strictly monotonic if $z \succ w$ for all $z \geq w$ with $z-w \neq 0$. 


\subsection{Preliminaries}

The assumptions from Debreu's 1962 article [6;Ch.10] are listed below with minor alterations. The conditions stated as (a.1) and (d.2) are equivalent in locally compact spaces such as Euclidean spaces to the conditions Debreu actually stated: $A(X) \bigcap-A(X)=\{0\}$ and $A(X) \bigcap A(Y)=\{0\}$, and imply them in general, as proved in the Appendix. The non-satiation assumption (b.1) of Debreu is strengthened to local non-satiation here. We take some linear topology $\tau$ for the choice space $L$, hopefully natural to the economic setting. For conciseness the following nine assumptions are collectively referred to as the Debreu conditions:

(a.1) $X \bigcap(-X)$ is bounded ${ }^{9}$;

for every $i \in M$ :

(a.2) $X_{i}$ is closed and convex;

(b.1) $\forall x_{i} \in \hat{X}_{i}$ and any neighborhood $\mathcal{N}$ of $x_{i}$ there exists $x_{i}^{\prime} \in \mathcal{N}$ such that $x_{i}^{\prime} \succ_{i} x_{i}$ closed;

(b.2) $\forall x_{i}^{\prime} \in X_{i}$, the sets $\left\{x_{i} \in X_{i}: x_{i} \succeq_{i} x_{i}^{\prime}\right\}$ and $\left\{x_{i} \in X_{i}: x_{i}^{\prime} \succeq_{i} x_{i}\right\}$ are

(b.3) for every $x_{i}^{\prime}$ in $X_{i}$ the set $\left\{x_{i} \in X_{i}: x_{i} \succeq_{i} x_{i}^{\prime}\right\}$ is convex;

(c.1) $(\{\omega\}+Y) \bigcap X \neq \emptyset$;

(c.2) there is a closed convex augmented total production set $\ddot{Y}$ such that

$$
\left(\left\{\omega_{i}\right\}+A(\ddot{Y})-D\right) \bigcap X_{i} \neq \emptyset \quad \forall i \in M
$$

for every $j \in N$ :

(d.1) $0 \in Y_{j} ;$ and

(d.2) $X \bigcap(Y+\{\omega\})$ is bounded.

We will need to add an additional topological assumption akin to the "Closedness Hypothesis" assumed by Mas-Colell [11]. The intent is to ensure that the set of feasible allocations, which is bounded under the Debreu conditions, can be captured in some compact set. This substitutes for the Heine-Borel property ${ }^{10}$ of $R^{n}$.

Capturing Hypothesis: There exists some linear topology $\mathcal{T}$ on $L$ such that $\tau$ bounded subsets of $L$ are relatively $\mathcal{T}$-compact and the following sets are $\mathcal{T}$-closed:

(i) $\ddot{Y}$,

(ii) $X_{i} \quad \forall i \in M$, and

(iii) $\left\{x_{i} \in X_{i}: x_{i} \succeq_{i} x_{i}^{\prime}\right\} \quad \forall x_{i}^{\prime} \in X_{i}, \quad \forall i \in M$.

9 A set in a locally convex space is bounded if absorbed by every zero neighborhood. A set $A$ absorbs a set $B$ in a real vector space if there is some scalar $\alpha>0$ such that $B \subset \beta A$ for all $\beta \geq \alpha$.

10 A topological vector space has the Heine-Borel property if each of its closed bounded subsets is compact. 
Remark 4.1 A sufficient condition for the Capturing Hypothesis is that $L$ is a semi-reflexive ${ }^{11}$ space. A space is semi-reflexive if and only if its bounded sets are relatively weak ${ }^{12}$ compact [15;p.144]. Thus the weak topology would be our choice for $\mathcal{T}$. It is a particularly nice choice since the closure of a convex set is the same for all locally convex topologies on $L$ consistent with its duality [15;p.130], including the weak topology. Then requiring the above sets (i)-(ii)-(iii) to be $\mathcal{T}$-closed would be superfluous, since they are all closed and convex in the original topology $\tau$ under the Debreu conditions. With Banach spaces, we can widen the class that is amenable to the Capturing Hypothesis from reflexive Banach spaces by including any conjugate ${ }^{13}$ Banach space, using Alaoglu's Theorem to place a topology on $L$ in which the unit ball is compact, the weak-star ${ }^{14}$ topology. In that case, denoting the pre-dual of $L$ by $V$, the minimum closedness restriction on the above sets would be Mackey ${ }^{15}-\langle L, V\rangle$ closed, since that is the finest topology consistent with a given duality. If the Banach space is reflexive, the Capturing Hypothesis is then entirely superfluous. Examples of reflexive Banach spaces are $L^{q}(\mu), 1<q<\infty$ (even if $\mu$ is not $\sigma$-finite). As an aside, the Dixmier-Goldberg-Ruston Theorem [8;23A] states necessary and sufficient condition for a Banach space to be a conjugate space or isomorphic to one. Of course any Montel ${ }^{16}$ space meets the Capturing Hypothesis in its "own original" topology by definition. Example Montel spaces are various types of spaces of infinitely differentiable functions and Schwarz distribution spaces [15]. Obviously any Euclidean space is a Montel space. This ends the remark.

We need a preliminary lemma to show that the Debreu conditions, which ensure that the feasible consumption and total production sets are compact in a Euclidean space, do roughly the same job here. Define $G \subset L^{m+1}$ by

$$
G=\left\{z \in L^{m+1}: \omega+z_{m+1}=\sum_{i=1}^{m} z_{i}\right\}
$$

Then any feasible allocation $(x, y) \in L^{m+n}$, where $y_{T}=\sum_{j=1}^{n} y_{j}$, satisfies $\left(x, y_{T}\right) \in$ $G$. Whenever the Capturing Hypothesis is in effect, we will use $\mathcal{T}^{k}$ to denote the implied $\mathcal{T}$-product topology on $L^{k}$, for any positive integer $k$.

11 A locally convex space $L$ is semi-reflexive if $L=\left(L^{*}\right)^{*}$. A number of alternative characterizations are given by Schaefer [15;p.144]. A reflexive space is a barrelled (e.g. normed) semi-reflexive space.

${ }^{12}$ For any duality $\left\langle L, L^{*}\right\rangle$ the weak topology on $L$, denoted $\sigma-\left\langle L, L^{*}\right\rangle$, is defined as the weakest topology preserving the duality.

13 A vector space is a conjugate space if it is dual to some other vector space.

14 If $L=V^{*}$, for some other space $V$, the weak-star topology on $L$ is the $\sigma-\langle L, V\rangle$ topology.

15 For a duality $\left\langle E, E^{*}\right\rangle$, the Mackey topology is that of uniform convergence on the set of all absolutely convex $\sigma-\left\langle E^{*}, E\right\rangle$ compact subsets of $E^{*}$. This is the finest topology on $E$ that is consistent with the duality $\left\langle E, E^{*}\right\rangle$.

16 A Montel space is a topological vector space with the Heine-Borel property, that is, in which any closed bounded subset is compact. 
Lemma 4.1. Suppose $\mathcal{E}$ is an economy on a locally convex choice space $L$. If $\mathcal{E}$ satisfies Debreu conditions (a.1), (a.2), (c.2), and (d.2), as well as the Capturing Hypothesis, then the set of feasible consumption-total production allocations, $\prod_{i=1}^{m} \hat{X}_{i} \times \hat{Y}=\left(\prod_{i=1}^{m} X_{i} \times Y\right) \bigcap G$, is compact in $\mathcal{T}^{m+1}$.

PRoOF: To show that $G$ is $\mathcal{T}^{m+1}$-closed, suppose, for some net directed by $(\Lambda, \geq)$, that $z^{\lambda} \rightarrow z \in L^{m+1}$ (in $\mathcal{T}^{m+1}$ ), where $z^{\lambda} \in G \quad \forall \lambda \in \Lambda$. Then

$$
v^{\lambda} \equiv z_{m+1}^{\lambda}-\sum_{i=1}^{m} z_{i}^{\lambda} \rightarrow v \equiv z_{m+1}-\sum_{i=1}^{m} z_{i}
$$

from the continuity of addition in $(L, \mathcal{T})$. But $v^{\lambda} \in\{-\omega\}$, a closed compact set in any linear topology, for all $\lambda \in \Lambda$, so $v \in\{-\omega\}$, implying $z \in G$.

The product of closed sets is closed in the product topology. Since $G$ is $\mathcal{T}^{m+1}$ closed and the intersection of closed sets is closed, it suffices to show that the projections of this product set $\hat{X}_{1}, \ldots, \hat{X}_{m}$ and $\hat{Y}$ are each bounded. For then each would be $\mathcal{T}$-closed and therefore $\mathcal{T}$-compact under the Capturing Hypothesis. By condition (d.2), $\hat{Y}$, the feasible total production set and $\hat{X}$, the feasible total consumption set, are bounded. Suppose $\hat{X}_{k}$ is not bounded for some $k \in M$. Then, since $\hat{X}$ is bounded, $\hat{X}_{k} \bigcap\left(-\sum_{i \neq k}^{m} X_{i}\right)$ must be unbounded, which contradicts (a.1).

\subsection{The Main Theorem}

Although the following theorem is stated for an extremely large class of topological vector spaces, the Capturing Hypothesis places a significant restriction on this class, or alternatively, on smoothness properties of agents' preferences and consumption sets. The proof is along the lines of Bewley [4; Theorem 1], starting with quasiequilibria on finite-dimensional subspaces of $L$ and extending along a net. The statement includes Debreu's theorem [6;Ch.10] as a special case. Let $\hat{D}$ denote the smallest cone containing the preference generated set

$$
\hat{\mathcal{D}}=\left\{z \in L: z+\omega_{i} \succ_{i} x_{i} \quad \forall x_{i} \in \hat{X}_{i}, \forall i \in M\right\} .
$$

Theorem 4.1. Suppose $\mathcal{E}$ is an economy on a locally convex choice space $L$ that satisfies the Debreu Conditions and Capturing Hypothesis. If $L$ is finite-dimensional or the augmented production set $\ddot{Y}$ can be chosen so that $A(\ddot{Y})-\hat{D}$ has interior, then $\mathcal{E}$ has a quasi-equilibrium.

Proof: By Lemma 4.1, for each agent $i, \hat{X}_{i}$ is $\mathcal{T}$-compact. Thus, given the Capturing Hypothesis, $\hat{X}_{i}$ has a maximal element for $\succeq_{i}$, which implies by condition (b.1) that there exists $v_{i} \in X_{i}$ such that $v_{i} \succ_{i} z$ for all $z \in \hat{X}_{i}$.

Let $\omega+\bar{y} \in(\{\omega\}+Y) \bigcap X$ (condition (c.1)), where $\bar{y}=\sum_{j=1}^{n} \bar{y}_{j}, \bar{y}_{j} \in Y_{j} \quad \forall j \in$ $N$, and let $\bar{x}_{i}=\omega_{i}+\sum_{j=1}^{n} \theta_{i j} \bar{y}_{j} \quad \forall i \in M$. Next, by condition (c.2), let

$$
\hat{x}_{i} \in\left(\left\{\omega_{i}\right\}+A(\ddot{Y})-D\right) \bigcap X_{i} \quad \forall i \in M .
$$


Let $\mathcal{F}$ be the set of finite dimensional subspaces of $L$ containing the vectors: $\omega_{i}, \bar{x}_{i}, \hat{x}_{i}, v_{i}, \quad \forall i \in M ; \bar{y}_{j} \quad \forall j \in N$; and $\hat{y} \in \operatorname{int}[A(\ddot{Y})-\hat{D}]$. For any $F \in \mathcal{F}$, let $\mathcal{E}^{F}$ be the economy

$$
\left(\left(X_{i} \bigcap F, \succeq_{i}^{F}\right) ;\left(Y_{j} \bigcap F\right) ;\left(\omega_{i}\right) ;\left(\theta_{i j}\right)\right),
$$

where $\succeq_{i}^{F}$ is the preference ordering induced on $X_{i}^{F}=X_{i} \bigcap F$ by $\succeq_{i}$. Let $D_{F}$ denote the cone generated by preferences in $\mathcal{E}^{F}$, defined analogously to the cone $D$ for $\mathcal{E}$, and note that $\hat{D} \cap F \subset D_{F}$. Take the augmented total production set for $\mathcal{E}^{F}$ to be $\ddot{Y}_{F}=\ddot{Y} \cap F$. One can quickly verify that each of the Debreu [6;Ch.10,p.136] conditions holds for $\mathcal{E}^{F}$, making use of Appendix Lemma A.5. Since the topology induced on any finite-dimensional subspace of a locally convex space is Euclidean, Debreu's 1962 theorem applies and $\mathcal{E}^{F}$ has a quasi-equilibrium $\left(x^{F}, y^{F}, p^{F}\right)$, where $\left(x^{F}, y^{F}\right) \in F^{m+n}$ and $p^{F} \in F^{*}$. Let $y_{T}^{F}=\sum_{j=1}^{n} y_{j}^{F}$.

Let $\Theta$ denote the cone $A(\ddot{Y})-\hat{D}$. Since $p^{F} \in F^{*}$ is by Debreu's constructive proof a non-zero element of the polar of the cone $\Theta_{F}=A\left(\ddot{Y}_{F}\right)-D_{F}$ [6;Ch.10,p.122], and $\hat{y}$ is interior to this cone in the topology induced on $F$, we have $p^{F} \cdot \hat{y}<0$. Since $\Theta \bigcap F \subset \Theta_{F}$, we also have $p^{F} \in(\Theta \bigcap F)^{\circ}$. Without loss of generality, let $p^{F} \cdot \hat{y}=-1$. Under the ordering induced on $L$ by the cone $(-\Theta)$, the conditions of the Krein-Rutman Theorem [15;p.227] are satisfied and $p^{F}$ has a continuous linear extension to all of $L$, which we also denote by $p^{F} \in L^{*}$. Furthermore, since $(-\Theta)$ serves the role of the "positive cone" in the Krein-Rutman Theorem, $p^{F} \in \Theta^{\circ}$. Since $\hat{y} \in \operatorname{int}(\Theta)$, we know $\hat{y} \in(\mathcal{N}+\{\hat{y}\}) \subset \Theta$, where $\mathcal{N}$ is some closed absolutely convex zero-neighborhood whose polar $\mathcal{N}^{\circ}$ is weak-star compact by the Alaoglu-Bourbaki Theorem [8;p.70]. Since $p^{F} \cdot z \leq 0 \quad \forall z \in(\mathcal{N}+\{\hat{y}\})$ and $p^{F} \cdot \hat{y}=-1$, we have $p^{F} \in \mathcal{N}^{\circ} \quad \forall F \in \mathcal{F}$.

By the preceding lemma the consumption allocation $x$ and total production allocation $\left(x^{F}, y_{T}^{F}\right)$ are elements of a fixed $\mathcal{T}^{m+1}$-compact subset of $L^{m+1 n}$ for all $F \in \mathcal{F}$. The set $\mathcal{F}$ is directed by inclusion so that the triples $\left(x^{F}, y_{T}^{F}, p^{F}\right), F \in \mathcal{F}$ can be placed in a net directed by this inclusion. Thus there is a convergent (in $\left.\mathcal{T}^{m+1} \times \sigma\left\langle L^{*}, L\right\rangle\right)$ subnet $\left(x^{\lambda}, y_{T}^{\lambda}, p^{\lambda}\right)$ directed by $\lambda \in(\Lambda, \geq)$, with limit $\left(x, y_{T}, p\right)$. Since $-1=p^{\lambda} \cdot \hat{y} \rightarrow p \cdot \hat{y}$, we know $p \neq 0$. Of course, given Lemma 4.1 , the limit is a feasible consumption and total production allocation, with $x_{i}$ in $X_{i}$ for each $i \in M$ and $y_{T}$ in $Y$. Then there exists $y_{j} \in Y_{j}$ for each $j \in N$ such that $y_{T}=\sum_{j=1}^{n} y_{j}$, and $(x, y) \in L^{m+n}$ is a feasible allocation. We will show that $(x, y, p)$ satisfies the remaining properties of a quasi-equilibrium. Here the proof follows the end of Bewley's proof closely.

First we claim that, for any feasible production allocation $(\hat{y})$ and any $x^{\prime} \succ_{i} x_{i}$, that

$$
p \cdot x^{\prime} \geq p \cdot\left(\omega_{i}+\sum_{j=1}^{n} \theta_{i j} \hat{y}_{j}\right) .
$$

Let $F(\lambda)$ denote the subspace corresponding to the element $\lambda$ of the net. By the definition of the net, the definition of equilibrium on the subspaces, and by the 
Capturing Hypothesis, there exists $\lambda^{\prime} \in \Lambda$ such that, for all $\lambda \geq \lambda^{\prime}$, the vectors $x^{\prime}, \hat{y}_{1}, \ldots, \hat{y}_{n}$ are in $F(\lambda)$ and

$$
p^{\lambda} \cdot x^{\prime}>p^{\lambda} \cdot x_{i}^{\lambda}=p^{\lambda} \cdot\left(\omega_{i}+\sum_{j=1}^{n} \theta_{i j} y_{j}^{\lambda}\right) \geq p^{\lambda} \cdot\left(\omega_{i}+\sum_{j=1}^{n} \theta_{i j} \hat{y}_{j}\right) .
$$

Since $\left\{p^{\lambda}\right\}$ converges weakly, we have (4.2). Now suppose $x^{\prime} \succeq_{i} x_{i}$. By the local non-satiation assumption (b.1), for any neighborhood $\mathcal{N}$ of $x^{\prime}$ there exists $x^{\prime \prime}$ in $\mathcal{N}$ such that $x^{\prime \prime} \succ_{i} x^{\prime}$, implying $p \cdot x^{\prime \prime} \geq p \cdot\left[\omega_{i}+\sum_{j=1}^{n} \theta_{i j} \hat{y}_{j}\right]$ by (4.2). Since $\mathcal{N}$ is arbitrary, (4.2) applies to any $x^{\prime} \succeq_{i} x_{i}$. In particular, we have

$$
p \cdot x_{i} \geq p \cdot\left[\omega_{i}+\sum_{j=1}^{n} \theta_{i j} y_{j}\right]
$$

for each agent $i$. Since $\sum_{i} x_{i}-\omega_{i}=\sum_{j} y_{j}$ and $\sum_{i} \theta_{i j}=1$ for each $j$, we then have $p \cdot x_{i}=p \cdot\left[\omega_{i}+\sum_{j} \theta_{i j} y_{j}\right]$ for all $i$. The conclusion of the previous paragraph thus yields

$$
z \succeq_{i} x_{i} \quad \Rightarrow \quad p \cdot z \geq p \cdot x_{i} \quad \forall z \in X_{i}, \quad \forall i \in M .
$$

For any firm $j \in N$ and any $z \in Y_{j}$, we can apply (4.2) to $x^{\prime}=x_{i}$ for each $i$ and $\hat{y}_{k}=y_{k}$ for $k \neq j, \hat{y}_{j}=z$. This shows that $p \cdot z \leq p \cdot y_{j}$, implying that $y_{j}$ maximizes market value for each firm $j$. Combining this with (4.3) shows that $(x, y, p)$ is a compensated equilibrium.

For a quasi-equilibrium it remains to show that one of (2.2) or (2.3) must hold. Suppose (2.3) does not hold and $z \in X_{i}$ is such that $p \cdot z<p \cdot x_{i}$. Now suppose $x^{\prime} \in X_{i}$ satisfies $p \cdot x^{\prime}=p \cdot x_{i}$ and $x^{\prime} \succ_{i} x_{i}$. Then, for any $\alpha \in[0,1)$ we know $x^{\alpha}=\alpha x^{\prime}+(1-\alpha) z$ satisfies $p \cdot x^{\alpha}<p \cdot x_{i}$. But by continuity of preferences (b.2) there exists $\alpha \in[0,1)$ such that $x^{\alpha} \succ_{i} x_{i}$, a contradiction of (4.3). Thus (2.2) must hold.

For technical reasons, this theorem assumes that the cone $A(\ddot{Y})-\hat{D}$ has interior, rather than non-empty interior for the cone $A(\ddot{Y})-D$ that contains it. Nothing in the spirit of things, however, prevents one from adopting the weaker assumption that $A(\ddot{Y})-D$ has interior, at least under additional regularity conditions, such as those of the following proposition. These additional conditions are effectively null for exchange economies.

Proposition 4.1. Suppose $\mathcal{E}$ is an economy on a locally convex choice space satisfying the Debreu conditions and Capturing Hypothesis. If the augmented production set $\ddot{Y}$ can be chosen so that $A(\ddot{Y})-D$ has interior, then $\mathcal{E}$ has a quasiequilibrium under the additional conditions: $X \cap(\ddot{Y}-D+\{\omega\})$ is bounded and $Y$ is closed and convex.

PRoOF: Let $C$ be any closed convex cone with interior contained by $A(\ddot{Y})-D$ and let $\Theta=A(\ddot{Y})+C$. Let $\tilde{\mathcal{E}}$ denote the economy identical to $\mathcal{E}$ except that $Y_{j}$ is replace 
by $\tilde{Y}_{j}=Y_{j}+\Theta$ for all $j \in N$. Let the augmented production set for $\tilde{\mathcal{E}}$ be its own total production set $Y+\Theta$. By the previous theorem, $\tilde{\mathcal{E}}$ has a quasi-equilibrium $(x, y, p)$. The total production vector $y_{T}=\sum_{j} y_{j}$ is of the form $y^{*}+y^{\prime}-\delta$, where $y^{*} \in Y, y^{\prime}-\delta \in \Theta, y^{\prime} \in A(\ddot{Y})$, and $\delta \in D$.

Since $y_{T}$ maximizes profits on $Y+\Theta$ relative to $p$, so do $y^{*}$ on $Y,\left(y^{\prime}-\delta\right)$ on $\Theta$, and $y^{\prime}$ on $A(\ddot{Y})$. Since $\Theta$ and $A(\ddot{Y})$ are cones, $p \cdot y^{\prime}=p \cdot\left(y^{\prime}-\delta\right)=0$, implying $p \cdot \delta=0$.

As $\delta \in D$, it has the form $\delta=\lambda \sum_{i}\left(x_{i}^{\prime}-\omega_{i}\right)$ for some scalar $\lambda \geq 0$ and $x_{i}^{\prime} \succ_{i} \hat{X}_{i}$, (the feasible set $\hat{X}_{i}$ refers to the original economy $\mathcal{E}$ ), for all $i \in M$. By Debreu's Lemma 7 [5; Chapter 10], $x_{i}^{\prime} \succ_{i} x_{i}$ for all $i \in M$. Along the lines of Debreu [5; pp. 143-144] there are two cases to check.

Case 1: For some $k \in M, p \cdot x_{k}>\inf \left\{p \cdot z ; z \in X_{k}\right\}$. Then $p \cdot \sum_{i} x_{i}^{\prime}>p \cdot\left(\omega+y_{T}\right)$, implying $p \cdot \sum_{i}\left(x_{i}^{\prime}-\omega_{i}\right)>p \cdot y_{T} \geq 0$. But then $p \cdot \delta=0$ implies $\lambda=0$, or $\delta=0$. Thus $y_{T}=y^{*}+y^{\prime} \in Y+A(\ddot{Y})$, implying $y_{T} \in Y$. Let $y^{\prime}=\left(y_{1}^{\prime}, \ldots, y_{n}^{\prime}\right), \quad y_{j}^{\prime} \in Y_{j}$, be any production allocation such that $\sum_{j} y_{j}^{\prime}=y_{T}$. Since $p \cdot y_{T} \geq p \cdot z$ for all $z \in Y$ and and $p \cdot y_{j} \geq p \cdot z$ for all $z \in Y_{j}$ for all $j \in N$, we have $p \cdot y_{j}=p \cdot y_{j}^{\prime}$, and $\left(x, y^{\prime}, p\right)$ is a quasi-equilibrium for $\mathcal{E}$.

Case 2: Suppose, on the other hand, $p \cdot x_{i}=\min p \cdot X_{i}$ for all $i \in M$. In this case $p \cdot \sum_{i} x_{i}=\min p \cdot X$ and $p \cdot y_{T}=\max p \cdot(Y-\Theta)=\sup p \cdot Y$. Thus the hyperplane $H$ generated by $p$ separates $X$ and $\{\omega\}+Y$. Let $(\check{x}, \check{y})$ be any feasible allocation for $\mathcal{E}$. Since $\sum_{i} \check{x}_{i}=\omega+\sum_{j} \check{y}_{j} \in H$, we know $p \cdot \sum_{i} \breve{x}_{i}=\min p \cdot X$ and $p \cdot \sum_{j}=\max p \cdot Y$. This implies that $p \cdot \check{x}_{i}=p \cdot x_{i}$ for all $i \in M$ and $p \cdot \check{y}_{j}=p \cdot y_{j}$ for all $j \in N$. Thus $(\check{x}, \check{y}, p)$ is a quasi-equilibrium for $\mathcal{E}$.

Remark 4.2: Variations on these conditions will work. For example, any convex cone generated by a bounded subset of $\mathcal{D}$ can be substituted for $\hat{D}$ in the interior condition of Theorem 4.1. The proof that a quasi-equilibrium exists is straightforward.

Remark 4.3: The non-empty interior conditions of Theorem 4.1 and Proposition 4.1 can be weakened to non-empty Mackey-interior, since the Mackey topology is consistent with the given duality. No changes in the proofs are necessary. This weakening is not important in many applications since the original topology is often given by a metric, in which case it coincides with the Mackey topology [15].

Remark 4.4: The (weak) convexity of preferences assumption (b.3) might be strengthened to convexity:

$\left(b .3^{\prime}\right)$ If $x^{\prime} \succ_{i} x$ then for all $\alpha \in(0,1), \quad \alpha x^{\prime}+(1-\alpha) x \succ_{i} x$.

Then the other hypotheses of Theorem 4.1 also ensure the existence of compensated equilibria. In fact, the compensated and quasi- equilibrium concepts are equivalent under these strengthened assumptions. See the proofs of Debreu $[5 ; 4.9(1)-(2)]$ that also apply here. For future reference the yet stronger assumption of strong convexity of preferences is stated:

$\left(b .3^{\prime \prime}\right)$ If $x^{\prime} \succeq_{i} x$ and $x^{\prime} \neq x$, then for all $\alpha \in(0,1), \quad \alpha x^{\prime}+(1-\alpha) x \succ_{i} x$. 
Under continuous preferences, the implications $\left(b .3^{\prime \prime}\right) \Rightarrow\left(b .3^{\prime}\right) \Rightarrow(b .3)$ are standard facts $[5]$.

Remark 4.5: It will be noted that we did not actually use the property that the sets $\left\{x \in X_{i}: z \succeq_{i} x\right\}$ are closed for each $z$ in $X_{i}$, for each agent $i$. In fact, we used only the property that such sets are lineally closed ${ }^{17}$. Thus the assumption of continuous preferences can be relaxed correspondingly.

There are many ways to argue that a quasi-equilibrium is in fact an equilibrium. Zame [18] has given several of these arguments, which are generally based on the nature of the choice space.

\subsection{Proper Preferences and Quasi-equilibria}

As promised, an easy extension of Mas-Colell's Theorem of quasi-equilibrium existence based on proper preferences is stated below for reference.

TheOREM 4.2. Suppose $\mathcal{E}$ is an exchange economy on a locally convex vector lattice $L$, the Capturing Hypothesis applies, and for all $i \in M$ :

(i) $X_{i}=L_{+}$,

(ii) $\omega_{i} \in L_{+}$, and

(iii) $\succeq_{i}$ is monotonic, continuous (b.2), convex $\left(b .3^{\prime}\right)$, and $v_{i}-$ proper for some $v_{i} \in L_{+}$.

Then $\mathcal{E}$ has both compensated equilibria and quasi-equilibria.

As remarked earlier, compensated equilibria and quasi-equilibria are the same under these assumptions. For simplicity, convex preferences $\left(b .3^{\prime}\right)$ are assumed here, rather than Mas-Colell's weaker assumption of weak convexity (b.3) plus strict monotonicity in the direction of $v_{i}$ and $\omega$. Mas-Colell assumed that $\omega \neq 0$, but of course if $\omega=0$ the trivially zero allocation and any non-zero $p \in L^{\prime}$ form an equilibrium in that case.

PROOF: Theorem 4.2 is proved by Mas-Colell [11], with two minor changes. The Alaoglu-Bourbaki Theorem on the weak-star compactness of polars of a zeroneighborhood is substituted for Alaoglu's Theorem on the weak-star compactness of the unit ball of a conjugate Banach space. The continuity of the price functional $p$ generating the separating hyperplane in Proposition VII is ensured by direct application of the separating hyperplane theorem [8], which guarantees that the separating hyperplane is closed, or that $p$ is continuous. The continuity of positive linear functionals on Banach lattices need not be invoked.

Remark 4.6: There are many ways to argue that a quasi-equilibrium is in fact an equilibrium. Zame [18] has given several of these arguments, which depend critically on the nature of the choice space and the pattern of agents' endowments. For brevity, we will merely record that if $(x, y, p)$ is a quasi-equilibrium, then it is also an equilibrium provided

$$
p \cdot x_{i} \neq \inf \left\{p \cdot z: z \in X_{i}\right\} \quad \forall i \in M .
$$

17 A subset $S$ of a vector space is lineally closed if $x$ is in $S$ whenever $\{\alpha x+(1-$ $\alpha) z ; \alpha \in[0,1)\} \subset S$ for $z$ in $S$. 
For if (4.2) applies then the following four conditions constitute a contradiction under the assumptions of Theorem 4.1: (i) $\hat{x}_{i} \in X_{i}$, (ii) $p \cdot \hat{x}_{i}<p \cdot x_{i}$, (iii) $z \succ_{i} x_{i}$, and (iv) $p \cdot z=p \cdot x_{i}$. That is, by assumption (b.2), for some $\alpha \in(0,1)$, we have $\alpha z+(1-\alpha) \hat{x}_{i}$, but then $p \cdot\left(\alpha z+(1-\alpha) \hat{x}_{i}\right)<p \cdot x_{i}$, contrary to the support properties of $p$.

\section{Price Support of Efficient Allocations}

This section addresses the existence of a price vector supporting an efficient allocation. The conditions are considerably weaker than those of the quasi-equilibrium existence theorem. The proposition is topology-free, applying an algebraic form of the separating hyperplane theorem, relying on a non-empty intrinsic core ${ }^{18}$ condition rather than a non-empty interior condition. The non-empty core condition is weaker than non-empty interior and in any case is topology-free. The further weakening to non-empty intrinsic core will mainly be of value for economies on a proper subspace of the choice space.

Proposition 5.1. If $(x, y) \in L^{m+n}$ is an efficient allocation for an economy $\mathcal{E}$ then $(x, y)$ can be supported by some $p \in L^{\prime}$ under the following conditions:

(i) $X^{*} \equiv \sum_{i=1}^{m}\left\{z \in X_{i}: z \succeq_{i} x_{i}\right\}$ is convex,

(ii) $Y$ is convex,

(iii) for some $k \in M$, there exists $v_{k} \in L$ such that

$$
x_{k}^{\prime}+\alpha v_{k} \succ_{k} x_{k}^{\prime} \quad \forall \alpha \in(0,1) \quad \forall x_{k}^{\prime} \in\left\{z \in \hat{X}_{k}: z \succeq_{k} x_{k}\right\}
$$

(iv) $Z \equiv X^{*}-Y-\{\omega\}$ has non-empty intrinsic core or $L$ is finite-dimensional. PROOF: First, 0 is not in the intrinsic core of $Z$ since $(x, y)$ is efficient, for otherwise, for some $\alpha \in(0,1)$, we would have $-\alpha v_{k} \in Z$, implying the existence of a feasible allocation:

$$
\left(x_{1}^{*}, \ldots, x_{k-1}^{*}, x_{k}^{*}+\alpha v_{k}, x_{k+1}^{*}, \ldots, x_{m}^{*}, y^{*}\right) \in L^{m+n},
$$

where $x_{i}^{*} \succeq_{i} x_{i} \quad \forall i \in M$. But $x_{k}^{*}+\alpha v_{k} \succ_{k} x_{k}^{*} \succeq_{k} x_{k}$, which would imply that $(x, y)$ is not efficient.

Thus $Z$ and $\{0\}$, both being convex, can be separated in the linear hull $H$ of $Z$ by a hyperplane generated by a non-zero linear functional $\hat{p} \in H^{\prime}$ [8;p.15], which can be extended to $p \in L^{\prime}$ [8;p.3]. That is, $p \cdot z \geq 0 \quad \forall z \in Z$.

To see that $p$ supports $(x, y)$, suppose $x_{k}^{\prime} \succeq_{k} x_{k}$ for any $k \in M$. Since $\sum_{i=1}^{m} x_{i} \in$ $(Y+\{\omega\})$ and $\left(x_{k}^{\prime}+\sum_{i \neq k} x_{i}\right) \in X^{*}$, we have $x_{k}^{\prime}-x_{k} \in Z$, so $p \cdot x_{k}^{\prime} \geq p \cdot x_{k}$. This is true for all $k \in M$. By a similar argument, $p \cdot y_{j}^{\prime} \leq p \cdot y_{j} \quad \forall y_{j}^{\prime} \in Y_{j}, \quad \forall j \in N$.

18 The core of a subset $W$ of a vector space $L$ consists of all points $w \in W$ such that, for each $z \neq w$ there exists $z^{\prime} \in(w, z)$ such that the interval $\left[w, z^{\prime}\right]$ is contained by $W$. The intrinsic core of $W$ is the core of $W$ relative to the affine hull of $W$. 
Remark 5.1: Any weakly efficient allocation can be supported under the same hypothesis provided condition (iii) in the statement of the proposition is strengthened to apply to all $i \in M$. The proof is almost identical, so omitted.

Remark 5.2: This proposition remains true when condition (iv) in its statement is weakened to:

$\left(i^{\prime}\right)$ There exists a convex cone $\Gamma \subset L$ such that $\Gamma \bigcap Z=\emptyset$ and $(Z-\Gamma)$ has intrinsic core, or $L$ is finite-dimensional.

The proof is almost identical to the one given, using the fact that $p \cdot z \geq 0 \quad \forall z \in$ $(Z-\Gamma)$ implies $p \in \Gamma^{\circ}$ since $\Gamma$ is a cone.

For a continuous supporting price functional, of course, the usual non-empty interior condition of the Hahn-Banach theorem can be assumed, as in Debreu [6; Chapter 5]. As in Remark 4.3 we can weaken this by assuming only Mackey interior. Any positive linear functional on a Banach lattice is continuous [15]. For such spaces, for example $L^{p}$ spaces, even the algebraic form of the supporting price functional given by Proposition 5.1 is thus continuous with monotonic preferences or free disposal, either of which guarantee positivity.

\section{Example: A Storage-Release Production Technology}

Here we illustrate an economy for which an equilibrium can be demonstrated precisely thanks to the theory just developed. We pick the choice space $L=R \times$ $L^{2}([0,1])$, under the product norm topology given by

$$
\|(s, r)\|_{L}=\left(s^{2}+\|r\|_{L^{2}([0,1])}^{2}\right)^{\frac{1}{2}} \quad \forall(s, r) \in R \times L^{2}([0,1]) .
$$

The space $R$ is interpreted as initial stocks of a commodity which can be stored and released for later consumption at a rate given by a square-integrable function of the unit time interval (an element of $L^{2}([0,1])$ ).

Specifically, the storage-release production technology is the production set

$$
Y_{S R}=\left\{(s, r) \in L: s+\int_{0}^{1} r^{+}(t) d t \leq 0\right\},
$$

where $r^{+}$denotes the positive part of the release rate $r$. That is, a feasible "netput" is one for which the total amount released is no more than the initial stock. This makes sense for economies with storable commodities; leisure time is a good counter-example. One could conceive, as an alternative justification, that there is an actual input-output technology admitting a similar production of non-storable commodities.

It is immediate that $Y_{S R}$ is itself a cone (closed under multiplication by positive scalars) and has interior. The latter property is evident for instance, from the fact that the ball of radius $\frac{1}{2}$ centered at $(-1,0)$ is contained by $Y_{S R}$, as quickly verified by a few calculations and the Cauchy-Schwarz inequality. ${ }^{19}$

19 Suppose $\|(-1,0)-(s, r)\|_{L} \leq 1 / 2$. Then $s \leq-1 / 2$ and $\|r\|_{L^{2}} \leq 1 / 2$, the latter implying $\int|r| \leq 1 / 2$ (by applying Cauchy-Schwarz with the function signum $[r(\cdot)])$, which means $(s, r) \in Y_{S R}$. 
Although free disposal is implicit in the definition of $Y_{S R}$, that can be avoided by the following device. Assume strictly monotonic preferences and, temporarily, add a free disposal firm $Y_{0}=-L_{+}$. Once an equilibrium is demonstrated, the chosen production from $Y_{0}$ is obviously zero, so the same equilibrium allocations and price vector are obviously an equilibrium for the economy without $Y_{0}$. This is essentially the concept behind the use of the cone $D$ in demonstrating quasi-equilibria.

Now we have the following simple corollaries.

Proposition 6.1. Suppose $\mathcal{E}$ is an economy on $L=R \times L^{2}([0,1]), Y$ contains $Y_{S R}$, and the Debreu conditions apply. Then $\mathcal{E}$ has a quasi-equilibrium.

PROOF: Since $L$ is a reflexive Banach space, the Capturing Hypothesis is superfluous (Remark 4.1). Of course $A(Y)$ contains $A\left(Y_{S R}\right)$, so both have interior. Then Theorem 4.1 applies.

For the next proposition let $\omega_{i R}$ denote the projection of $\omega_{i}$ from $L$ into $R$, the amount of initial stored commodity endowed to agent $i$. We now have a simple and complete set of equilibrium conditions for this economy that are easily generalized.

Proposition 6.2. Suppose $L=R \times L^{2}([0,1])$ and $Y=Y_{S R}$. Then $\mathcal{E}$ has an equilibrium under the following conditions, for all $i \in M$ :

(1) positive consumption $\left(X_{i}=L_{+}\right)$,

(2) $\succeq_{i}$ is non-satiated (b.1), continuous (b.2), and weakly convex (b.3), and

(3) storage endowments $\left(\omega_{i} \geq 0\right.$ and $\left.\omega_{i R}>0\right)$.

PRoOF: The Debreu conditions (a.1) and (a.2) are implied by (1); (c.1) and (c.2) by (1) and (3); and (d.1) and (d.2) by (1) and the definition of $Y_{S R}$. The remaining conditions for the statement of Theorem 4.1 are satisfied as in Proposition 6.1. Finally condition (2.3) can be ruled out by the definition of $Y_{S R}$, which implies the existence for each agent of some $x_{i}$ in $X_{i}$ such that $x_{i}-\omega_{i} \in \operatorname{int}[A(Y)-D]$. For this point, see Debreu [6, page 146].

The same approach can be used in a two period setting under uncertainty. One assumes that there exists some scalar $\alpha>0$ such that a fixed large quantity of deterministic first period input can be converted in some production technology to any output whose positive part has expected value smaller than $\alpha$. The assumption is more onerous in this case, however. The same storage-release idea, perhaps combined with an actual production input-output technology can be quite plausibly applied to sequence choice spaces. The first element of an agent's endowed sequence vector would represent that agent's initial "capital stock".

\section{Concluding Remarks}

This paper has in part been an exploratory study of the alternative properties of infinite-dimensional spaces that make equilibrium analysis tractable. We will review. As explained, a positive cone with interior is very helpful. It makes our extra assumption that the production set contains a price block even weaker than free disposal. In this regard, and in the generalization of consumption sets and 
bounding restrictions, this paper generalizes the equilibrium existence theorems of Bewley [4] and Magill [10] along roughly the same lines that Debreu [6;Ch.10] generalizes Arrow and Debreu [1].

This interior property aside, the "nicest" commodity spaces seem to be separable reflexive Banach lattices. Separability (along with the norm topology) "buys" the existence of utility representations for preferences. In conjunction with the Banach lattice property, separability also ensures that the positive cone has a quasiinterior, which makes the formulation of conditions ensuring equilibrium existence easier (See Zame [18] for details on the definition and importance of quasi-interior). This latter property is also shared by any of the spaces $L^{q}(\mu) 1 \leq q \leq \infty$, separable or not, (provided the underlying measure space is totally $\sigma$-finite.) Reflexivity makes superfluous the Capturing Hypothesis, a substitute for the local compactness of finite-dimensional spaces that crops up throughout this paper. In general, the ability to place some topology on the choice space in which feasible allocations are captured in a compact set seems imperative. The decomposition property of lattices [15] is important in the construction of a preference based price block [11].

Mas-Colell [11] includes a fairly complete list of the literature on equilibria in infinite-dimensional choice spaces up to the time that this paper first appeared in 1983. Since then, however, several related papers have appeared. Mas-Colell's approach has been extended to general proper binary orders for preferences in a recent paper by Yannelis and Zame [17]. Jones [9] demonstrated equilibrium with production in a Banach space roughly along the lines of this paper. Zame [18] demonstrated equilibrium with production on a normed vector lattice by following the basic outline of Mas-Colell forming conditions on the "marginal efficiency" of production. Again, consumption sets are the positive cone. Although Zame's production conditions are somewhat complicated, they definitely seem less restrictive that assuming that the recession cone of the production set has interior, at least when the positive cone of the space itself has no interior. Certainly the current state of the art is not yet satisfactory in so far as posing simple and generally applicable conditions for the existence of equilibria in a range of vector spaces.

The original version of this paper includes several supplementary results that have been left out for brevity. These include sufficient conditions for the conclusions: a quasi-equilibrium is an equilibrium, a valuation equilibrium has an efficient allocation, an exchange equilibrium has an efficient allocation, there exist efficient allocations, and an allocation in the core of the allocation game of all replications of an economy is an equilibrium.

\section{Appendix - Recession Cones in General Spaces}

This appendix is devoted to extending certain properties of recession cones stated by Debreu [5] for Euclidean spaces. Debreu's definition of recession cone applies to arbitrary sets in Euclidean spaces. The definition we give below applies to convex sets in arbitrary real vector spaces. Beer [3] examines the extension to non-convex sets, along with a different economic application. The recession cone, $A(X)$, of a convex subset $X$ of a real vector space $L$ is the set $\{z \in L:\{z\}+X \subset X\}$. Recession cones are also known as "asymptotic cones" or "characteristic cones". If 
one follows the convention that a "cone" $C$ must satisfy $C \bigcap-C=\{0\}$ (that is, $C$ cannot be a nontrivial vector subspace), then recession cones are not actually cones, but rather "wedges", sets closed under multiplication by positive scalars. There does not seem to be a standard reference on recession cones for general vector spaces, although Holmes [8] has a few results. I have thus collected together the following properties, which are almost immediate from the definition above.

Lemma A.1. Suppose $L$ is a vector space over the field of real numbers.

(i) If $X$ is a convex subset of $L$, then $A(X)$ is convex.

(ii) If $X$ is a convex subset of $L$, then $A(X+\{z\})=A(X) \quad \forall z \in L$.

(iii) If $X$ is a convex subset of $L$ and $0 \in X$, then $A(X) \subset X$.

(iv) If $X$ and $Y$ are convex subsets of $L$, then $A(X) \subset A(X+Y)$.

(v) If $X_{i}, i=1, \ldots, m$ are convex subsets of $L$, then

$$
A\left(\prod_{i=1}^{m} X_{i}\right) \subset \prod_{i=1}^{m} A\left(X_{i}\right) .
$$

The following lemma is from Holmes [8;p.34] and Beer [3].

Lemma A.2. Suppose $X$ is a convex subset of a real vector space $L$. Then

$$
A(X)=\left\{z \in L: x+\alpha z \in X \quad \forall \alpha \in R_{+}, \quad \forall x \in X\right\} .
$$

If, in addition, $L$ is a topological vector space in which $X$ is closed, then, for any $x \in X$,

$$
A(X)=\left\{z \in L: x+\alpha z \in X \quad \forall \alpha \in R_{+}\right\} .
$$

Lemma A.3. Suppose $X$ is a closed convex subset of a real topological vector space. Then $A(X)$ is closed.

Proof: By Lemma A.2, for any $x \in X$,

$$
A(X)=\bigcap_{\alpha \in R_{++}}\{\alpha(X-\{x\})\} .
$$

Since this is the intersection of closed sets, $A(X)$ is closed.

Lemma A.4. If $X$ is the non-empty intersection of an arbitrary collection of closed convex subsets $\left\{X_{\lambda}: \lambda \in \Lambda\right\}$ of a real topological vector space, then

$$
A(X)=\bigcap_{\lambda \in \Lambda} A\left(X_{\lambda}\right)
$$

PRoOF: If $x \in X$ then $z \in A(X)$ if and only if

$$
\left\{x+\alpha z: \alpha \in R_{+}\right\} \subset X_{\lambda} \quad \forall \lambda \in \Lambda,
$$

which implies $z \in A\left(X_{\lambda}\right) \quad \forall \lambda \in \Lambda$, by Lemma A.2. 
The following lemma shows the connection between the boundedness of the intersection of two convex sets and the trivial intersection of their recession cones.

Lemma A.5. Suppose $X$ and $Y$ are closed convex subsets of a real topological vector space $L$. If $X \cap Y$ is bounded then $A(X) \cap A(Y)=\{0\}$. If, furthermore, $L$ is a locally compact ${ }^{20}$ then the converse is also true; indeed $A(X) \cap A(Y)=\{0\}$ then implies that $X \cap Y$ is compact.

PROOF: From Lemma A.4, the first assertion follows if the recession cone of a bounded set is $\{0\}$; but this is obvious.

For the second assertion, let $U$ be a compact balanced zero neighborhood of $L$. From Lemma A.4 the conclusion follows if $A(X)=\{0\}$ implies $X \subset(\alpha U)$, for some positive scalar $\alpha$, since $X$ would then be a closed subset of a compact set, and therefore compact. Suppose this is not the case, and define

$$
I_{n}=X \bigcap(L \backslash(n U)) \neq \emptyset \quad \forall n \in \mathbf{N}
$$

where $\mathbf{N}$ is the set of positive integers. Define

$$
C=X \bigcap(\bar{n} U) \bigcap\left(L \backslash \operatorname{int}\left(\frac{\bar{n}}{2} U\right)\right)
$$

where $\bar{n}$ is an integer sufficiently large to ensure $C \neq \emptyset$. Note that $C$ is compact. Let $B_{n}=\left\{\alpha I_{n}: \alpha \in R_{+}\right\} \cap C$. Assume, without loss of generality (Lemma A.1), that $0 \in X$. The sequence of sets $\left(B_{n}\right)_{n=1}^{\infty}$ must have a non-zero cluster point $z \in C$ since $C$ is compact. Choose any $x \in X$ and any $\alpha \in R_{+}$. If we can show $x+\alpha z \in X$, then we are done, since $z \in A(X)$ is a contradiction.

Choose $\hat{n} \in \mathbf{N}$ such that $\alpha z \in \operatorname{int}(\hat{n} U)$. Let $W$ be any balanced zero-neighborhood. For any scalar $\gamma>0$, and any integer $n>\hat{n}$, there exists a scalar $\delta>0$ and some $u \in \gamma W$ such that $w \equiv \delta(\alpha z+u) \in I_{n} \subset X$, with $\alpha z+u \in(\hat{n} U)$. This implies that $\delta \geq n / \hat{n}$. Now

$$
x+\alpha z=x+\frac{w}{\delta}-u=x+\frac{(w-x)}{\delta}+\frac{x}{\delta}-u .
$$

Since $X$ is convex and since $n>\hat{n}$ implies $\delta>1$, we know $x+(w-x) / \delta \in X$. We can take $\delta$ so large that $x / \delta \in(\gamma W)$. Thus $x+\alpha z \in X+(2 \gamma W)$, but since $\gamma$ is arbitrarily small, $x+\alpha z \in \bar{X}=X$, since $X$ is closed.

I do not know of a larger class of locally convex spaces with the property that $A(X)=\{0\}$ implies that $X$ is bounded. Reflexive Banach spaces may be a suitably general class. For our purposes this is mainly of historical interest, relating to Debreu's original assumptions. The latter condition seems more intuitive and convenient for its purpose in competitive analysis.

20 A topological vector space is locally compact if every point has a compact neighborhood. An example is Euclidean space. 


\section{References}

K.J. Arrow and G. Debreu, 1954, Existence of equilibria for a competitive economy, Econometrica 22, 265-290.

K.J. Arrow and F.H. Hahn, 1971, General Competitive Analysis, (San Francisco: Holden-Day).

G. Beer, 1979, Recession Cones of Nonconvex Sets and Increasing Functions, Proceedings of the American Mathematical Society 73, 228-232.

T. Bewley, 1972, Existence of equilibria in economies with infinitely many commodities, Journal of Economic Theory 4, 514-540.

G. Debreu, 1959, Theory of Value, (Cowles Foundation Monograph 17, Yale University Press, New Haven and London). (Cambridge University Press).

M. Florenzano, 1982, On the existence of equilibria in economies with an infinitedimensional commodity space, Research Paper 8217, CNRS-CEPREMAP Paris, France.

R. Holmes, 1975, Geometric Functional Analysis and Its Applications, (SpringerVerlag, New York).

L. Jones, 1982, Existence of Equilibrium with Infinitely Many Consumers and Infinitely Many Commodities, Research Paper , Northwestern University.

M.J.P. Magill, 1981, An Equilibrium Existence Theorem, Journal of Mathematical Analysis and Applications 84, 162-169.

A. Mas-Colell, 1983, The price equilibrium existence problem in Banach Lattices, Harvard University (mimeo).

L.W. McKenzie, 1959, On the Existence of General Equilibrium for a Competitive Market, Econometrica 27, .

, 1961, On The Existence of General Equilibrium: Some Corrections, Econometrica 29, 247-248.

W. Rudin, 1973, Functional Analysis, (McGraw Hill, New York).

H.H. Schaefer, 1971, Topological Vector Spaces, (New York: Springer-Verlag).

, 1974, Banach Lattices and Positive Operators, (Berlin, New York: Springer-Verlag).

N.C. Yannelis and W.R. Zame, 1984, Equilibria in Banach Lattices Without Ordered Preferences, IMA Reprint Series No. 71, Institute for Mathematics and its Applications, University of Minnesota.

W. Zame, 1985, Equilibria in Production Economies with an Infinite-Dimensional Commodity Space, IMA Reprint Series Number 127, Institute for Mathematics and its Applications, University of Minnesota. 